Document downloaded from:

http://hdl.handle.net/10251/54873

This paper must be cited as:

Ballester Bolinches, A.; Beidleman, JC.; Cossey, J.; Esteban Romero, R.; Rangland, MF.; Schmidt, J. (2009). Permutable subnormal subgroups of finite groups. Archiv der Mathematik. 92(6):549-557. doi:10.1007/s00013-009-2976-x.

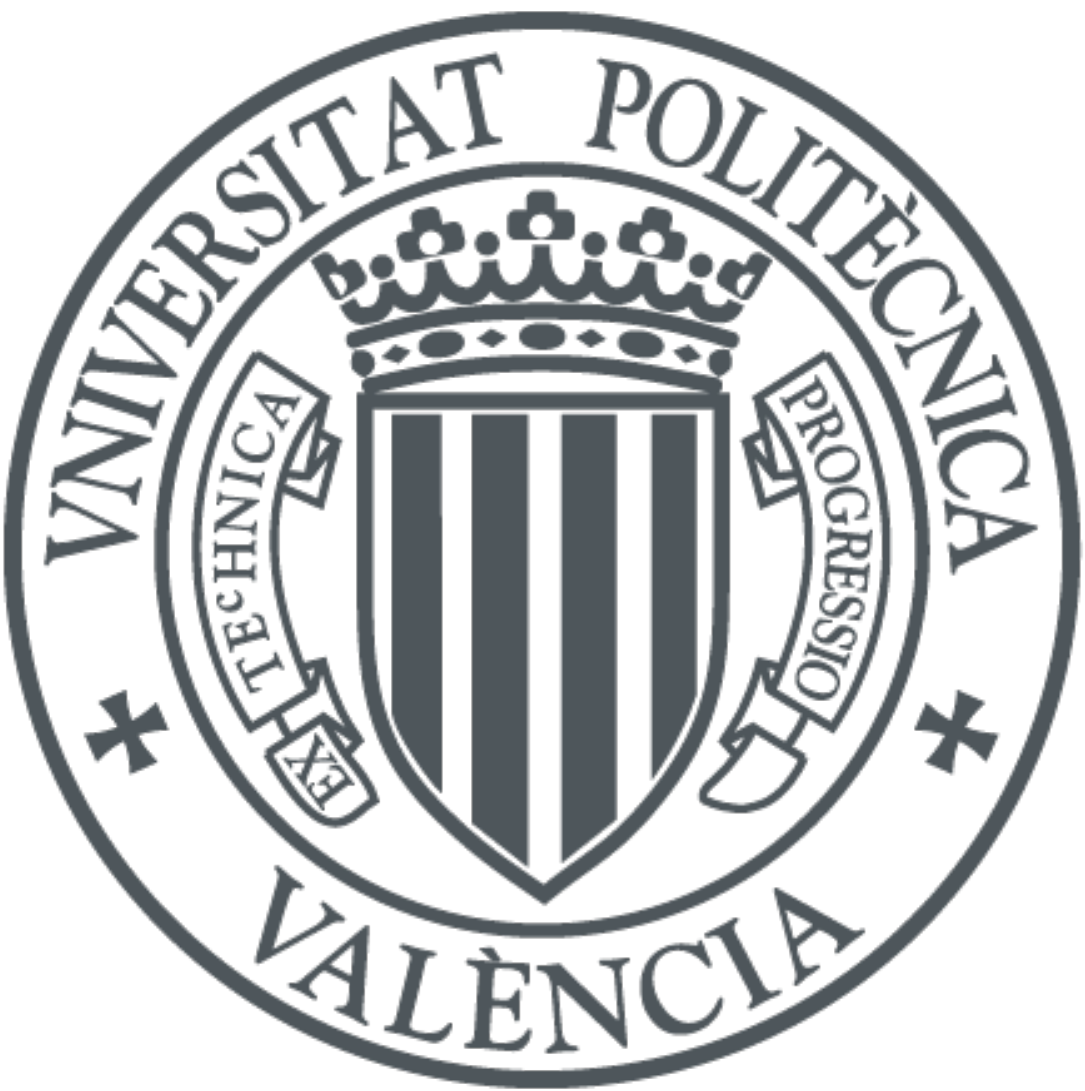

The final publication is available at

http://link.springer.com/article/10.1007\%2Fs00013-009-2976-X

Copyright Springer Verlag (Germany)

Additional Information

The final publication is available at Springer via http://dx.doi.org/10.1007/s00013-009-2976-x 


\title{
PERMUTABLE SUBNORMAL SUBGROUPS OF FINITE GROUPS
}

\author{
A. BALLESTER-BOLINCHES, J. C. BEIDLEMAN, JOHN COSSEY, \\ R. ESTEBAN-ROMERO, M. F. RAGLAND, AND JACK SCHMIDT
}

\begin{abstract}
The aim of this paper is to prove certain characterization theorems for groups in which permutability is a transitive relation, the so called $\mathcal{P} \mathcal{T}$-groups. In particular, it is shown that the finite solvable $\mathcal{P} \mathcal{T}$-groups, the finite solvable groups in which every subnormal subgroup of defect two is permutable, the finite solvable groups in which every normal subgroup is permutable sensitive, and the finite solvable groups in which conjugate-permutability and permutability coincide are all one and the same class. This follows from our main result which says that the finite modular $p$-groups, $p$ a prime, are those $p$-groups in which every subnormal subgroup of defect two is permutable or, equivalently, in which every normal subgroup is permutable sensitive. However, there exist finite insolvable groups which are not $\mathcal{P} \mathcal{T}$-groups but all subnormal subgroups of defect two are permutable.
\end{abstract}

\section{INTRODUCTION}

All groups considered are finite. All unexplained notation and terminology can be found in [4] or [9]. A subgroup $K$ of $G$ is said to be permutable (S-permutable) in $G$ provided $K H=H K$ for all subgroups (Sylow subgroups) $H$ of $G$. A well known result of Ore [8] shows that permutable subgroups are necessarily subnormal. Kegel [6] generalized this result showing that S-permutable subgroups are subnormal. A group $G$ is called a $\mathcal{T}$-group provided normality is a transitive relation, that is, $H \unlhd K \unlhd G$ implies $H \unlhd G$. Similarly, one defines $\mathcal{P} \mathcal{T}$-groups and $\mathcal{P S T}$ groups as those groups in which, respectively, permutability and S-permutability are transitive relations. As a consequence of the results of Ore and Kegel, one has that the $\mathcal{P} \mathcal{T}$-groups, respectively $\mathcal{P S} \mathcal{S}$-groups, are those groups in which permutability, respectively S-permutability, coincides with subnormality.

The classes $\mathcal{T}, \mathcal{P T}$, and $\mathcal{P S T}$ have been studied in a number of articles with much of the work done in the past 10 years (see [3] for long list of references). In particular, the first, fourth, and fifth authors determined in [2] that the groups in which every subnormal subgroup of defect two is S-permutable are precisely the $\mathcal{P S} \mathcal{T}$-groups. Let us record this theorem for reference.

Theorem 1 (Ballester-Bolinches, Esteban-Romero, and Ragland [2]). The groups satisfying the property

$$
H \unlhd K \unlhd G \text { implies } H \text { is } S \text {-permutable in } G
$$

Date: August 1, 2015.

1991 Mathematics Subject Classification. Primary 20D10, 20D20, 20 D35.

Key words and phrases. Permutable, Subnormal, $\mathcal{P} \mathcal{T}$-group, Conjugate-Permutable, Modular $p$-group. 
1 are precisely the $\mathcal{P S} \mathcal{S}$-groups.

$2 \quad$ A natural question to ask is if a similar statement holds for $\mathcal{P} \mathcal{T}$-groups. That 3 is, are the $\mathcal{P} \mathcal{T}$-groups precisely the groups $G$ for which $H \unlhd K \unlhd G$ implies $H$ is 4 permutable in $G$ ? One aim of this article is to give an affirmative answer to this 5 question in the solvable universe. However, we provide a counterexample which 6 shows that the answer is negative for insolvable groups.

The second and fifth authors introduced the concepts of permutable sensitivity and S-permutable sensitivity in [3].

9 Definition 2. A subgroup $H$ of a group $G$ is said to be

(1) permutable sensitive in $G$ if the following holds:

$\{N \mid N$ is permutable in $H\}=\{H \cap W \mid W$ is permutable in $G\}$.

(2) S-permutable sensitive in $G$ if the following holds:

$\{N \mid N$ is S-permutable in $H\}=\{H \cap W \mid W$ is S-permutable in $G\}$.

It was shown in [3] that the groups whose normal subgroups are S-permutable sensitive are the $\mathcal{P} \mathcal{S} \mathcal{T}$-groups and that the groups whose subnormal subgroups are permutable sensitive are the $\mathcal{P} \mathcal{T}$-groups.

Theorem 3 (Beidleman and Ragland [3]). The groups whose normal subgroups are $S$-permutable sensitive are precisely the $\mathcal{P S} \mathcal{S}$-groups.

It was left as an open question there whether or not the $\mathcal{P} \mathcal{T}$-groups could be thought of as the groups whose normal subgroups are permutable sensitive. Another aim of this article is to answer this open question in the affirmative in the solvable universe.

Let us now recall the concept of conjugate-permutability.

Definition 4. A subgroup $H \leq G$ is said to be conjugate-permutable in $G$ if $H H^{g}=H^{g} H$ for all $g$ in $G$, that is, $H$ permutes with all of its conjugates in $G$.

An old result of Szép [11] (see also [5, Corollary 1.1]) generalizing Ore's aforementioned result shows that every conjugate-permutable subgroup is subnormal. The converse obviously holds for subnormal subgroups of defect two.

We will show in the solvable universe that a slightly stronger property for conjugate-permutable subgroups is equivalent to being a $\mathcal{P} \mathcal{T}$-group. That is, a solvable group $G$ is a $\mathcal{P} \mathcal{T}$-group if and only if every conjugate-permutable subgroup of $G$ is permutable in $G$.

The answers to the already mentioned questions are contingent on what happens in the $p$-group case, $p$ a prime. In fact, the main result of the paper provides a new characterization of modular $p$-groups.

Theorem A. For a p-group $G$ the following statements are equivalent.

(i) $G$ has modular subgroup lattice;

(ii) $G$ has all subnormal subgroups of defect two permutable;

(iii) $G$ has all normal subgroups permutable sensitive.

(iv) $G$ has all conjugate-permutable subgroups permutable. 


\section{Preliminaries}

In this section, we introduce some terminology, notation, and some needed results not discussed in the introduction.

Let $p$ be a prime. It is well-known that the modular $p$-groups are exactly those $p$-groups with all subgroups permutable. The following three results on modularity and $p$-groups will be essential in the proofs of our main results. First we recall the classic result of Iwasawa (see Theorem 2.3.1 of [9]) on the characterization of modular $p$-groups.

\section{Theorem 5. A p-group $G$ is modular if and only if}

(i) $G$ is a direct product of a quaternion group $Q_{8}$ of order 8 with an elementary abelian 2-group, or

(ii) $G$ contains an abelian normal subgroup $A$ with cyclic factor group $G / A$; further there exists an element $b \in G$ with $G=A \rtimes\langle b\rangle$ and a positive integer $s$ such that $a^{b}=a^{1+p^{s}}$ for all $a \in A$, with $s \geq 2$ in case $p=2$.

The following can be found as Lemmas 2.3.3 and 2.3.4 of [9].

Lemma 6. A p-group $G$ is not modular if and only if there exists a section $H / K$ of $G$ with $H / K$ isomorphic to the dihedral group of order 8 or the non-abelian group of order $p^{3}$ and exponent $p$ for $p>2$.

Lemma 7. Let $G$ be a p-group with an abelian subgroup $A$ such that every subgroup of $A$ is normal in $G$ and $G / A$ is cyclic. For $p=2$ and $A$ of exponent greater than 2, further assume that there exists subgroups $A_{2} \leq A_{1} \leq A$ with $A_{1} / A_{2}$ cyclic of order 4 and $\left[A_{1}, G\right] \leq A_{2}$. Then $G$ is a modular p-group.

The following lemma can be found as Proposizione 1.6 of [7]

Lemma 8. Let $G$ be a non-modular p-group all of whose proper factor groups are modular. Then $G$ has a unique minimal normal subgroup.

Our next theorem contains classical results of Agrawal's from [1] characterizing solvable $\mathcal{P} \mathcal{S} \mathcal{T}$-groups and $\mathcal{P} \mathcal{T}$-groups.

Theorem 9 (Agrawal [1]).

(i) $G$ is a solvable $\mathcal{P S T}$-group if and only if the nilpotent residual of $G$ is an abelian Hall subgroup of $G$ acted upon by conjugation as a group of power automorphisms by $G$.

(ii) $G$ is a solvable $\mathcal{P} \mathcal{T}$-group if and only if $G$ is a solvable $\mathcal{P S T}$-group with $G / L$ a $\mathcal{P} \mathcal{T}$-group.

\section{A New Characterization of Modular $p$-Groups}

As it is mentioned in the above section, the modular $p$-groups can be thought of as those $p$-groups all of whose subgroups are permutable. It is a natural question to ask if one can impose less permutability conditions on the subgroups of a $p$-group and not lose modularity. The answer to that question is contained in Theorem A. It shows that one only needs to require the subgroups of defect two in a $p$-group to be permutable in order for the group to enjoy the property of having a modular subgroup lattice.

For $p$ an odd prime, we let $M(p)$ denote the non-abelian group of order $p^{3}$ and exponent $p$. For $p=2$, we let $M(p)$ denote the dihedral group of order 8 . We 
1 let $\mathcal{X}$ denote the class of $p$-groups in which every subnormal subgroup of defect 2 two is permutable and we let $\mathcal{Y}$ denote the class of $p$-groups all of whose normal 3 subgroups are permutable sensitive. The class of modular $p$-groups will be denoted by $\mathcal{M}$. Note that the classes $\mathcal{X}$ and $\mathcal{Y}$ are quotient closed.

We need the following lemma.

Lemma A. Let $G$ be a p-group and suppose that one of the following two conditions hold.

(1) Every subnormal subgroup of $G$ of defect two is permutable in $G$.

(2) Every normal subgroup of $G$ is permutable sensitive in $G$.

In addition, assume $G$ has a unique minimal normal subgroup $N$ with $G / N$ modular but $G$ not modular. Then

(i) $G$ does not contain a normal subgroup $H$ isomorphic to $M(p)$;

(ii) $G / N$ is not abelian, and;

(iii) $G / N$ is not Hamiltonian.

15 Proof. (i) Suppose $G$ does contain $H$ as a normal subgroup with $H \simeq M(p)$. Then $N$ is contained in $H$.

Assume that $p=2$ and $H / N$ is not central in $G / N$. Then there is an element $g \in G$ and a non-central element $x \in H$ such that $\left\langle x, x^{g}\right\rangle=H$. Let $z=\left[x^{g}, x\right]$ and note that $x$ must have order 2 and $N=\langle z\rangle$. Since $G / N$ is modular, $\langle g, z\rangle$ is permutable in $G$ and hence $\langle x, g\rangle=\langle x\rangle\langle g, z\rangle$ so that $\langle g, z\rangle$ has index 2 in $\langle x, g\rangle$. In particular, $\langle g, z\rangle$ is a normal subgroup of $\langle x, g\rangle$. The group $\langle x N\rangle\langle g N\rangle$ is one of three possible semidirect products; $x N$ maps $g N$ to $g^{k} N$ where $k=-1, k=2^{n}-1$, or $k=2^{n}+1$ where $|g N|=2^{n+1}$. One can then deduce that $[g, x] \in\left\langle g^{2}, z\right\rangle$. Now $z$ is a power of $g$ since for some $i$ and $j$, we have $z=\left[x^{g}, x\right]=[g, x]^{2}=\left(g^{2 i} z^{j}\right)^{2}=g^{4 i}$. Note that $[g, x]$ has order 4 since $z=[g, x]^{2}$ has order 2. Also note that $\langle g\rangle$ has index 2 in $\langle x, g\rangle$ since $\langle g\rangle=\langle g, z\rangle$. Since $\langle g\rangle$ is normal in $\langle x, g\rangle, g^{x}=g^{t}$ with $t$ either $-1,2^{m}-1$, or $2^{m}+1$ where $|g|=2^{n+2}=2^{m+1}$. In these three cases, $[g, x]$ has order $2^{m}, 2^{m}$ and 2 , respectively. Hence $m=2$ and $g$ has order 8 . Thus $\langle x, g\rangle=\left\langle x, g \mid x^{2}=g^{8}=1,[g, x]=g^{s}\right\rangle$ where $s=2$ or $s=-2$. It is clear from the presentation of $\langle x, g\rangle$ that $\langle x N, g N\rangle$ is dihedral of order 8, a contradiction to Lemma 6. Consequently, $H / N$ is central in $G / N$. It means that every subgroup of $H$ is subnormal in $G$ of defect at most 2 . Hence if $G$ were an $\mathcal{X}$-group, $H$ would be a modular group. Therefore $G$ must be a $\mathcal{Y}$-group. Let $A$ be a subgroup of $H$. If $N$ is contained in $A$, then $A$ is normal in $G$. Assume that $N$ is not contained in $A$. Then $A N$ is normal in $G$. Since $A N$ is permutable sensitive and $A$ is normal in $A N$, there exists a permutable subgroup $K$ of $G$ such that $A N \cap K=A$. If $H \cap K$ is not contained in $A N$, it follows that $H=(A N)(H \cap K)=H \cap K$ and then $A N=A$. This contradiction yields $H \cap K \leq A N$ and so $A=H \cap K$ is permutable in $H$. Consequently, $H$ is a modular group. This contradicts Lemma 6 . Therefore $p$ is and odd prime. Then $H$ contains a normal elementary abelian subgroup of $G$, 41 say $L$, of order $p^{2}$. Let $x$ be an element of $L$ which is not central in $H$. Suppose first 42 that $G \in \mathcal{X}$. Then $\langle x\rangle$ a subnormal subgroup of defect two in $G$ and so permutable 43 in $G$ and hence in $H$. This is a contradiction. Now suppose $G \in \mathcal{Y}$. Then $\langle x\rangle$ 44 is normal in $L$ and since $L$ is permutable sensitive in $G,\langle x\rangle=L \cap M$ for some 45 subgroup $M$ permutable in $G$. But since $M \cap H$ contains $x$ and does not contain $N$, 46 we must have $M \cap H=\langle x\rangle$. Hence $\langle x\rangle$ is permutable in $H$. This final contradiction 47 proves statement (i). 
(ii) Suppose that $G / N$ is abelian and derive a contradiction. Let $E$ be an arbitrary subgroup of $G$. If $N \leq E$, then $E$ is normal in $G$. Suppose then that $E$ does not contain $N$. Then $E$ is normal in $N E$ and $N E$ is normal in $G$. So if $G \in \mathcal{X}$ we have $E$ is permutable in $G$. Hence $G$ is modular, contrary to the choice of $G$. Assume $G \in \mathcal{Y}$. Since $N$ is central, $N \leq C_{G}(E)$ so that $C_{G}(E)$ is normal in $G$. Thus $C_{G}(E)$ is permutable sensitive in $G$ and there is a permutable subgroup $K$ of $G$ with $K \cap C_{G}(E)=E$. If $K$ is not contained in $C_{G}(E)$ then $[E, K]=N$ and so $N \leq E K$. Thus if $n$ is a non-trivial element of $N$ we have $n=h k$ for some $h \in E$ and $k \in K$. But then $k=h^{-1} n \in C_{G}(E)$ and since $K \cap C_{G}(E)=E$ we have $k \in E$ and so $n \in E$, which contradicts the fact that $N$ is not contained in $E$. Hence every subgroup is permutable and $G$ is modular. This is the final contradiction.

(iii) Suppose that $G / N$ is Hamiltonian. Then $G / N$ is the direct product of an elementary abelian 2-group $E / N$ and the quaternion group $Q / N$ of order 8 . Since the quaternion group of order 8 has trivial Schur multiplier by Corollary 11.22 of [10], it follows that $Q=Q_{0} \times N$ with $Q_{0}$ non-abelian. But then $Q^{\prime}=Q_{0}^{\prime}$ is a normal subgroup of $G$ with $Q^{\prime} \cap N=1$, a contradiction.

Proof of Theorem $\boldsymbol{A}$. We are trying to show $\mathcal{X}=\mathcal{Y}=\mathcal{M}$. Clearly $\mathcal{M}$ is contained in $\mathcal{X} \cap \mathcal{Y}$ and so it is enough to show that $\mathcal{X}$ and $\mathcal{Y}$ are contained in $\mathcal{M}$.

Suppose $G \in \mathcal{X} \cup \mathcal{Y}$ with the order of $G$ minimal with respect to $G$ not being modular. By Lemma 8 we have $G$ possesses a unique minimal normal subgroup, say $N$. Now $G / N \in \mathcal{M}$ and by Lemma A parts (ii) and (iii), $G / N$ is not Dedekind. Hence, by Theorem $5, G / N=A / N \rtimes C N / N$ with $A / N$ abelian and $C N / N$ cyclic acting on $A / N$ as power automorphisms in the following way: for some positive integer $s,(a N)^{c N}=(a N)^{1+p^{s}}$ for all $a \in A$ where $C=\langle c\rangle$.

Suppose $\Phi(A)=1$. Then $(a N)^{c N}=(a N)^{1+p^{s}}=a N$. Hence $c N$ centralizes $A / N$. Thus $G / N=A / N \times C N / N$ is abelian, a contradiction to Lemma A part (ii). Thus we can assume $\Phi(A) \neq 1$.

Next suppose $A^{p}=1$. Then $A$ is not abelian or we would have $\Phi(A)=1$. Hence $p$ is odd. Let $x$ and $y$ be two non-commuting elements of $A$. Then $\langle x, y\rangle$ is a subgroup of $A$ containing $N$ which is isomorphic to $M(p)$. Hence $\langle x, y\rangle \unlhd G$. This contradicts Lemma A part (i). Hence $A^{p} \neq 1$.

Now $A / N$ is abelian so that $A^{\prime} \leq N \leq Z(A)$. Hence, for $a, b \in A,\left[a^{p}, b\right]=$ $[a, b]^{p}=1$ so that $A^{p} \leq Z(A)$. Moreover, for $a \in A$, we have $\left(a^{p}\right)^{c}=\left(a^{c}\right)^{p}=$ $\left(a^{1+p^{s}} n\right)^{p}$ where $n$ is some element of $N$. Hence $\left(a^{p}\right)^{c}=\left(a^{p}\right)^{1+p^{s}}$. So if $x \in A^{p}$ has order $p$, then $x$ is central in $G$. Hence $\Omega\left(A^{p}\right)=N$. But $A^{p}$ is abelian and thus $A^{p}$ is cyclic.

Let $y$ be of maximal order in $A$. Let us show that $A$ possesses a generating set $X$ with $y \in X$ and every other element of $X$ having order $p$. Since $y$ is of maximal order in $A,\left\langle y^{p}\right\rangle=A^{p}$. Let $\left\{a_{1}, a_{2}, \ldots, a_{n}, y\right\}$ be any generating set for $A$. For each $i$, write $a_{i}^{p}=y^{p t_{i}}$ where $t_{i}$ is some integer. Note that the identity

40 holds for all $a, b \in A$ since $A^{\prime} \leq Z(G)$.

${ }_{41}$ First suppose $p$ is odd. Now $\left(a_{i} y^{-t_{i}}\right)^{p}$ is easily seen to be trivial using equa42 tion $\left(^{*}\right)$. Let $b_{i}=a_{i} y^{-t_{i}}$. Since $\left\langle b_{i}, y\right\rangle=\left\langle a_{i}, y\right\rangle$, the set of non-trivial elements in $43\left\{b_{1}, b_{2}, \ldots, b_{n}, y\right\}$ is a generating set of the desired type.

$44 \quad$ Suppose now that $p=2$. Then $\left(a_{i} y^{-t_{i}}\right)^{2}=\left[y^{-t_{i}}, a_{i}\right]$ follows from equation $(*)$. 45 Let $b_{i}=a_{i} y^{-t_{i}}$. Since the order of $y$ is greater than 4 by Lemma A part (ii), it 
1 follows that $b_{i}^{2} \in N \leq A^{4}=\left\langle y^{4}\right\rangle$. Now, for each $i$, write $b_{i}^{2}=y^{4 s_{i}}$ where $s_{i}$ is some integer. Now write $c_{i}=b_{i} y^{-2 s_{i}}$. Equation $\left(^{*}\right)$ yields $c_{i}^{2}=\left[y^{-2 s_{i}}, b_{i}\right]$ and since $y^{-2 s_{i}} \in A^{2} \leq Z(A)$, we have that $c_{i}^{2}=1$. Since $\left\langle c_{i}, y\right\rangle=\left\langle b_{i}, y\right\rangle=\left\langle a_{i}, y\right\rangle$, the set of nontrivial elements in $\left\{c_{1}, c_{2}, \ldots, c_{n}, y\right\}$ is a generating set of the desired type.

Now let $\left\{a_{1}, a_{2}, \ldots, a_{n}, y\right\}$ be a generating set for $A$ with the order of each $a_{i}$ equal to $p$. If $\left\langle a_{i}, a_{j}\right\rangle$ is non-abelian, then $N \leq\left\langle a_{i}, a_{j}\right\rangle \simeq M(p)$, contradicting Lemma A part (i). Thus each $a_{i}$ and $a_{j}$ commute and we have $M=$ $\left\langle a_{1}, a_{2}, \ldots, a_{n}, N\right\rangle$ is an elementary abelian normal subgroup of $G$. Thus each subgroup of $M$ is subnormal of defect two in $G$. Hence if $G \in \mathcal{X}$ then every subgroup of $M$ is permutable in $G$. Suppose $G \in \mathcal{Y}$. Then the argument used in the proof of Lemma A part (ii) gives all subgroups of $M$ are permutable in $G$.

Suppose that $g \in G \backslash C_{G}(M)$. Then $[a, g] \neq 1$ for some $a \in M$. Note that $\langle a\rangle N \unlhd G$ and also note that $\langle g\rangle \unlhd\langle a\rangle\langle g\rangle$. Hence $1 \neq[a, g] \in\langle a\rangle N \cap\langle g\rangle$. Now if $N \not \leq\langle g\rangle$ then we have $\langle a\rangle \leq\langle a\rangle N=(\langle a\rangle N \cap\langle g\rangle) N \leq C_{G}(g)$, a contradiction to $[a, g] \neq 1$. Hence $N \leq\langle g\rangle$. Thus $\langle g\rangle$ is permutable in $G$ for all $g \in G \backslash C_{G}(M)$.

For a final contradiction, we are now left with showing that if $g, h \in C_{G}(M)$, then $\langle g\rangle\langle h\rangle=\langle h\rangle\langle g\rangle$ giving all subgroups of $G$ permutable. Let $D=\langle y\rangle C$. From Lemma 7 , it is clear that $D$ is a modular $p$-group when $p$ is odd. A little more explanation is needed for the case when $p=2$. With $2^{\alpha}$ denoting the order of $y$, let $A_{1}=\left\langle y^{2^{\alpha-3}}\right\rangle$ and $A_{2}=\left\langle y^{2^{\alpha-1}}\right\rangle=N$. Note that $\alpha \geq 3$ or else $\exp (A) \leq 4$ in which case it is easily deduced from its structure that $G / N$ is abelian, a contradiction to Lemma A part (ii). Hence $A_{1} / A_{2}$ is cyclic of order four and $\left[A_{1}, D\right] \leq A_{2}$. So we have $D$ is a modular $p$-group using Lemma 7 . Let $g, h \in C_{G}(M)$ and write $g=m u$ and $h=m^{\prime} v$ with $m, m^{\prime} \in M$ and $u, v \in D$. Note that $g, h \in C_{G}(M)$ implies $u, v \in C_{G}(M)$. Since $D$ is modular, $X=\langle u\rangle\langle v\rangle$ is a subgroup and $u v=v^{k} u^{l}$ for integers $k$ and $l$. We have $u^{1-l} \Phi(X)=v^{k-1} \Phi(X)$. If $X$ is not cyclic, then $\langle v \Phi(X)\rangle \cap\langle u \Phi(X)\rangle=1$ in which case $u^{1-l} \Phi(X)=v^{k-1} \Phi(X)$ gives rise to $k \equiv l \equiv 1$ $\bmod p$. If $X$ is cyclic, then $k=l=1$. Hence we have

$$
g h=m u m^{\prime} v=m m^{\prime} u v=m m^{\prime} v^{k} u^{l}=\left(m^{\prime}\right)^{k} v^{k} m^{l} v^{l}=\left(m^{\prime} v\right)^{k}(m u)^{l}=h^{k} g^{l} .
$$

Now $g h=h^{k} g^{l}$ implies $\langle g\rangle$ permutes with $\langle h\rangle$. So all subgroups of $G$ are permutable, a contradiction. Thus if $G \in \mathcal{X} \cup \mathcal{Y}$, then $G$ is modular.

Assume now that every conjugate-permutable subgroup of a group $G$ is permutable. Then $G \in \mathcal{X}=\mathcal{M}$.

\section{An Application}

As an application of Theorem A, we prove the following theorem.

Theorem B. The following statements are equivalent for a solvable group $G$.

(i) $G$ is a $\mathcal{P} \mathcal{T}$-group.

(ii) Every subnormal subgroup of defect two in $G$ is permutable in $G$.

(iii) Every normal subgroup of $G$ is permutable sensitive in $G$.

(iv) Every conjugate-permutable subgroup of $G$ is permutable in $G$.

Proof. Note that the properties of the statements (ii)-(iv) are quotient closed and every $\mathcal{P} \mathcal{T}$-group satisfies (ii)-(iv).

Assume now that a solvable group $G$ satisfies one of the conditions (ii)-(iv). We prove that $G$ is a $\mathcal{P} \mathcal{T}$-group by induction on $|G|$. Applying either Theorem 1 or Theorem $3, G$ is a solvable $\mathcal{P S T}$-group. Moreover, by Theorem A, we may assume 
1 that $G$ is not a $p$-group. Suppose that $G$ is nilpotent. Then all Sylow subgroups 2 of $G$ are modular and so $G$ is a solvable $\mathcal{P} \mathcal{T}$-group. Therefore we can suppose 3 that $G$ is not nilpotent. If $L$ is the nilpotent residual of $G$, then $G / L$ is a solvable $4 \mathcal{P} \mathcal{T}$-group and $G$ is a solvable $\mathcal{P} \mathcal{T}$-group by Theorem 9 .

\section{A Counterexample}

Finally we present an example of a group with all subnormal subgroups of defect two permutable which is not a $\mathcal{P} \mathcal{T}$-group.

Example. Let $A=\langle a\rangle$ be a cyclic group of order 27. Then $A$ has an automorphism $b$ of order 9 acting on $A$ as $a^{b}=a^{4}$. The corresponding semidirect product $M=[A]\langle b\rangle$ is an Iwasawa group. Note that the center of $G$ has order 3.

On the other hand, let $D=\mathrm{SL}_{3}(4)$. This group has center of order 3 . We can construct the central product $C$ of $D$ and $M$ in which both centers are identified.

Let $t$ be an automorphism of order 3 acting on $D$ as the inner automorphism induced by the matrix

$$
\left(\begin{array}{lll}
1 & 0 & 0 \\
0 & 1 & 0 \\
0 & 0 & \mu
\end{array}\right),
$$

where $\mu$ is a generator of the multiplicative group of $\mathrm{GF}(4)$, and on $M$ as the inner automorphism of $M$ induced by $a^{3}$. Since $t$ acts trivially on the centers of $D$ and $M, t$ induces an automorphism of $C$ of order 3 . We see that $D\langle t\rangle$ is isomorphic to $\mathrm{GL}_{3}(4)$. Let $G$ be the semidirect product $G=[C]\langle t\rangle$.

We see that $\mathrm{O}_{3}(G)=M$ is the solvable radical of $G$ and that $D$ is the solvable residual of $G$. On the other hand, every normal subgroup of $M$ is normal in $G$ because it is centralized by $D$ and normalized by $t$. Furthermore, all $3^{\prime}$-elements of $G$ centralize $M$. It follows that the normal closure $\left\langle H^{G}\right\rangle$ of a subgroup $H$ of $M$ in $G$ coincides with the normal closure $\left\langle H^{P}\right\rangle$ of $H$ in a Sylow 3-subgroup $P$ of $G$, and since $D$ centralizes $M$ and $t$ acts on $M$ as an inner automorphism, it coincides with the normal closure $\left\langle H^{M}\right\rangle$ of $H$ in $M$. We can check that every subnormal subgroup of $G$ of defect 2 is permutable in $G$ : For the solvable subnormal subgroups, which are contained in $M$, it is enough to check permutability in $P$, and these subgroups are conjugate to $\left\langle b, a^{9}\right\rangle$, to $\left\langle b a, a^{9}\right\rangle$, to $\left\langle b a^{2}, a^{9}\right\rangle$ or to $\left\langle b^{3}\right\rangle$. For an insolvable subgroup $H, H$ contains $D$ and since $G / D$ is a $\mathcal{P} \mathcal{T}$-group, $H / D$ is permutable in $G / D$ and so $H$ is permutable in $G$. Nevertheless, the subgroup $\langle b\rangle$ is a subnormal subgroup of $M$ (and so of $G$ ) of defect 3 which does not permute with $\langle t\rangle$, because $\langle b, t\rangle$ contains the element $b^{t}=b^{a^{3}}=b a^{-9}$ and so $a^{9} \in\langle b, t\rangle$.

\section{ACKNOWLEDGMENT}

This paper was started and completed in two visits of the first author to the Department of Mathematics of the Australian National University, Canberra, Australia. He is grateful to the Department of Mathematics for its warm hospitality and financial support while this investigation was carried out. He also gratefully acknowledges financial support by Grant MTM2007-68010-C03-02 from MEC (Spanish Government) and FEDER.

The research of the fourth author has been supported by the Grants MTM200768010-C03-02 from MEC (Spanish Government) and FEDER, and GV/2007/243 
1 from Generalitat (Valencian Government). He thanks all these institutions for their 2 financial support.

\section{REFERENCES}

[1] R. K. Agrawal, Finite groups whose subnormal subgroups permute with all Sylow subgroups, Proc. Amer. Math. Soc., 47 (1975), 77-83.

[2] A. Ballester-Bolinches, R. Esteban-Romero, and M. Ragland, A note on finite $\mathscr{P} \mathscr{S} \mathscr{T}$-groups, J. Group Theory, 10 no. 2, (2007), 205-210.

[3] J. C. Beidleman and M. F. Ragland, The intersection map of subgroups and certain classes of finite groups, Ric. Mat. (2007), 56 no. 2, 217-227.

[4] K. Doerk and T. Hawkes, Finite Soluble Groups, Walter De Gruyter, Berlin/New York, 1992.

[5] T. Foguel, Conjugate-permutable subgroups, J. Algebra, 191 (1997), no. 1, 235-239.

[6] O. Kegel, Sylow-Gruppen und Subnormalteiler endlicher Gruppen, Math. Z., 78, (1962), 205-221.

[7] P. Longobardi, Finite groups with modular factors, Note Mat., 2 (1982), no. 1, 73-100.

[8] O. Ore, Contributions to the theory of groups of finite order, Duke Math. J., 5 (1939), no. $2,431-460$.

[9] R. Schmidt, Subgroup lattices of groups Vol. 14, Walter de Gruyter \& Co., (1994).

[10] J. J. Rotman, An introduction to the theory of groups. Fourth Edition, Graduated Texts in Mathematics 119, Springer, New York (1995).

[11] J. Szép, Bemerkung zu einem Satz von O. Ore, Publ. Math. Debrecen, 3 (1953), 81-82.

A. Ballester-Bolinches (corresponding author), Departament D’Àlgebra, Universitat de València, Dr. Moliner, 50, E-46100 Burjassot, València, Spain

E-mail address: Adolfo.Ballester@uv.es

J. C. Beidleman, Department of Mathematics, 745 Patterson Office Tower, UniverSity of KentuCKy, LeXington, KY, 40506-0027, U.S.A.

E-mail address: clark@ms.uky.edu

John Cossey, Mathematics Department, Mathematical Sciences Institute, Australian National University, 0200, Canberra, Australia

E-mail address: John.Cossey@maths.anu.edu.au

R. Esteban-Romero, Institut Universitari de Matemàtica Pura i Aplicada, Universitat Politècnica de València, Camí de Vera, s/n, E-46022 València, Spain

E-mail address: resteban@mat.upv.es

M. F. Ragland, Department of Mathematics, Auburn University Montgomery, P.O. Box 244023, Montgomery, AL, 36124-4023, U.S.A.

E-mail address: mragland@aum.edu

Jack Schmidt, Department of Mathematics, 745 Patterson Office Tower, University of Kentucky, Lexington, KY, 40506-0027, U.S.A.

E-mail address: jack@ms.uky.edu 